\title{
Hábitos de vida, enfermidades e quedas referidas por idosos do ambiente rural
}

\author{
Lifestyle habits, illnesses and falls in the \\ elderly individuals' rural environment
}

FisiSenectus. Unochapecó Ano 1 - Edição especial - 2013 p. $20-28$

\section{Fatima Ferretti}

Professora do Programa de Mestrado em Ciências da Saúde da Unochapecó, Dr.a em Saúde Coletiva pela Unifesp, ferrettifisio@yahoo.com.br

\section{Leonardo Rinaldi}

Fisioterapeuta formado pela Unochapecó, leonardorinaldi2@yahoo.com.br

\section{Leticia Rinaldi}

Fisioterapeuta formados pela Unochapecó, lerinaldi@unochapeco.edu.br

\section{Cezar Grontowski Ribeiro}

Professor titular do curso de Educação Física no Instituto Federal do Paraná - campus Palmas, mestrando em Ciências da Saúde pela Unochapecó, prof.cezar2@yahoo.com.br

\section{Gisele Knebel Zoletti}

Acadêmica do $8^{\circ}$ período do curso de graduação em Fisioterapia da Unochapecó, gii.zoletti@unochapeco.edu.br

\section{Resumo}

Introdução: O envelhecimento humano é um processo biológico, social e psicológico que sofre influências do meio em que a pessoa vive, seja urbano, seja rural. Objetivo: Verificar os hábitos de vida, enfermidades e quedas referidas por idosos do ambiente rural. Metodologia: A pesquisa caracterizou-se como quantitativa e transversal com uma amostra de 260 idosos residentes em ambiente rural. Os instrumentos de coleta de dados foram um questionário sobre Condições de saúde adaptado de Morais ${ }^{9}$ e $\mathrm{MMSE}^{8}$. Resultados: Em relação aos hábitos, a atividade física mais praticada foi a caminhada (50,38\%); a maioria não faz uso de tabaco $(88,16 \%)$ e não ingere bebidas alcoólicas (71,92\%). Quanto às enfermidades autorreferidas destacam-se o déficit visual $(72,30 \%)$, os distúrbios osteomioarticulares (59,23\%), hipertensão (46,92\%) e insônia (41,53\%). No que tange à incidência de quedas, 76 idosos (29,23\%) relataram ter sofrido queda no último ano e 41 sofreram pequenas lesões derivadas do evento. Conclusão: 0 processo de envelhecer deixa o ser humano mais suscetível ao desenvolvimento de enfermidades crônicas e os hábitos de vida são estratégias fundamentais para a prevenção destes eventos. A sociedade e o poder público têm um papel importante no que tange ao planejamento de ações para atender estes sujeitos, respeitando suas particularidades e necessidades, de acordo com o meio onde vivem, no caso deste estudo, o território rural.

\section{Palavras-chave}

Envelhecimento. Idosos. Saúde. 


\begin{abstract}
Introduction: Human aging is a biological, social and psychological suffering influences the environment in which the person lives, whether urban or rural. Objective: To assess lifestyle habits, illnesses and falls in elderly individuals' rural environment. Methodology: The research was characterized as quantitative and cross-sectional sample of 260 older adults living in a rural environment. The instruments for data collection were a questionnaire on health conditions and adapted Morais9 MMSE8. Results: Regarding habits, physical activity was the most practiced walking (50.38\%), the majority does not use tobacco $(88.16 \%)$ and not ingest alcoholic beverages (71.92\%). Regarding self-reported illnesses highlight the visual deficit (72.30\%), musculoskeletal disorders (59.23\%), hypertension (46.92\%) and insomnia (41.53\%). Regarding the incidence of falls, 76 elderly $(29.23 \%)$ reported having been dropped in the last year and 41 suffered minor injuries derived from the event. Conclusion: The aging process leaves humans more susceptible to the development of chronic diseases and lifestyle are key strategies for preventing these events. Society and government has an important role regarding the planning of actions to meet these guys, respecting their particularities and needs, according to the environment where they live, in the case of this study, the rural territory.
\end{abstract}

\title{
Keywords
}

Aging. Elderly. Health

\section{Introdução}

$\diamond<\infty<\infty<\infty<\infty<\infty<\infty<\infty<\infty<\infty<\infty<\infty<\infty<\infty<\infty<\infty<\infty$

O envelhecimento humano é uma temática de estudo importante, em função de do aumento da população idosa, somado a ocorrência de eventos crônicos nessa fase ${ }^{1}$. Essa expansão da população idosa evidencia a necessidade de pesquisas que busquem conhecer as características, interesses, necessidades e, principalmente, vulnerabilidades desse grupo.

Envelhecer leva a diminuição das reservas funcionais do organismo, o que pode ser observado em todos os aparelhos e sistemas, de acordo com cada indivíduo $0^{2,3}$. É importante ressaltar que o contexto em que a pessoa nasce, cresce e enveIhece contribui, e muito, para a maneira de como se dá o processo de envelhecimento, sendo este heterogêneo e influenciado por várias dimensões. Os hábitos de vida do idoso correspondem a uma somatória de vivências passadas, cuidados com a saúde, condições de moradia, educação, alimentação e higiene, sejam no ambiente rural ou urbano, e impactam na qualidade da sua saúde ${ }^{4}$.

O meio rural destaca-se pela satisfação do idoso com a manutenção de algum tipo de trabalho, pois, diante de tantas mudanças, ele tem a oportunidade de continuar a realizar as atividades que desempenhou durante toda a vida. Esse meio também traz vantagens no que tange ao contexto físi- co, que contínua por mais tempo inalterado, com um ritmo de vida mais lento, favorecendo a relação e trocas sociais, proporcionando a manutenção dos laços afetivos com os que os rodeiam ${ }^{5}$.

0 município de Constantina, caso deste estudo, no norte do estado do Rio Grande do Sul, possui um total de 9.842 habitantes, sendo $15,10 \%$ com idade igual ou superior a 60 anos, o que destaca 0 município enquanto população longeva, por apresentar índice muito superior à média nacional, que é de $7,4 \%$ atualmente ${ }^{1}$.

Dentre eles, 817 são do sexo feminino e 670 do sexo masculino. Destes, 1.487 idosos, 874 reside em ambiente rural, totalizando $58,77 \%$ da população total nesta faixa etária, fator que chama a atenção, pois é um percentual significativo para esse território, quando comparado à média nacional, da região Sul e do estado do Rio Grande do Sul ${ }^{1}$.

Compreendendo que o contexto rural não tem se constituído como prioridade das pesquisas sobre o processo de envelhecer e que ter informações sobre as condições de saúde desse grupo é fundamental para planejar ações com o objetivo de promover um envelhecimento saudável daqueles que ainda hoje residem em meio rural, o presente estudo terá como objetivo verificar os hábitos de vida, enfermidades e quedas referidas por idosos do ambiente rural. 


\section{Materiais e métodos}

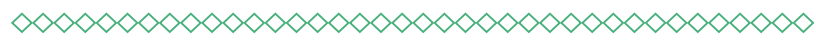

Pesquisa é quantitativa, de cunho descritivo transversal em que foram entrevistados 260 idosos de ambos os sexos, residentes em 11 comunidades rurais do município de Constantina (RS), com idade igual ou superior a 60 anos.

Os instrumentos de coleta de dados utilizados para esta pesquisa foram um questionário para identificar as condições de saúde dos idosos que residem no ambiente rural e o Exame Cognitivo Mini-Mental (MMSE) para verificar o cognitivo do idoso ${ }^{6}$.

Os critérios de inclusão utilizados foram possuir 60 ou mais anos de idade cronológica; ser residente no meio rural do município pesquisado; apresentar estado cognitivo preservado no MMSE, para o seu grau de escolaridade, conforme escore previsto no teste. 0 critério de exclusão foi idoso ausente em seu domicílio depois de três visitas.

Para a realização da coleta de dados foi realizada uma reunião com representantes da Unidade Básica de Saúde e da Assistência Social para informar-Ihes sobre a finalidade da pesquisa e solicitar o banco de dados com os endereços dos idosos para realizar a coleta no domicílio. De posse deste banco, a coleta foi realizada nas residências no período de janeiro e março do ano de 2010.

Nas visitas domiciliares os idosos receberam esclarecimentos sobre os objetivos, finalidades e a relevância do estudo e após serem convidados a participar da pesquisa, aqueles que aceitaram assinaram o termo de consentimento livre e esclarecido, conforme preconiza a resolução 196/96, que estabelece as diretrizes e normas reguladoras de pesquisa envolvendo seres humanos.

Em seguida aplicava-se o MMSE ${ }^{6}$, com objetivo de diagnosticar alterações do nível da função cognitiva. Quando verificado que o idoso apresentava estado cognitivo preservado, era aplicado o questionário de Condições de Saúde adaptado de Morais ${ }^{7}$. Esta pesquisa foi aprovada pelo comitê de ética da instituição sob o protocolo CE/277/2009.
A análise de dados foi realizada por meio da estatística descritiva e inferencial utilizando o Programa SPSS versão 17.0 .

\section{Resultados}

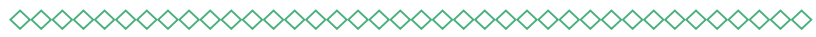

Quanto ao perfil dos entrevistados observou-se que a idade variou entre 60 a 94 anos, com média de $71,92( \pm 8,27)$ anos. Na distribuição por gênero, as mulheres predominaram sobre os homens sendo $56,53 \%$ do gênero feminino e $43,46 \%$ do masculino (Tabela 1 ). Com relação ao escore do MMSE, a média geral apresentou $21,05( \pm 1,72)$.

Em relação aos hábitos de vida, quando verificada a prática de atividade física, 50,38\% relataram que realizavam caminhada, $6,53 \%$ jogavam bocha e $42,30 \%$ não realizavam nenhum tipo de atividades físicas. Quanto à ingestão de bebidas alcoólicas, $71,92 \%$ relataram não fazer uso, enquanto $28,08 \%$ afirmaram beber com frequência. Dentre as bebidas mais ingeridas estão o vinho, em $80,92 \%$ dos casos, seguido da cerveja, com $18,30 \%$, e destilados, com 0,78\%. Em relação ao tabagismo, 88,84\% não possuíam o hábito de fumar e $11,16 \%$ fumavam ou fumaram em algum tempo da vida. Dentre os tipos de cigarros fumados pelos idosos são mais frequente os de maço $98,30 \%$, e 1,70\% de palha (Tabela 2).

Em relação às enfermidades autorreferidas pelos idosos, observou-se que $72,30 \%$ afirmam possuir problemas de visão, 59,23\% relataram ter DOMA (disfunções osteomioarticular), podendo ser osteoporose, osteoartrose ou artrite, 46,92\% apresentaram hipertensão arterial sistêmica, 41,53\% sofrem com insônia, 23,84\% tonturas, $21,15 \%$ relataram ter incontinência urinária quando sujeitos a esforço, 19,61\% declararam possuir dificuldades auditivas, $12,30 \%$ disseram possuir bronquite asmática, 9,23\% depressão, 3,84\% possuíam diagnóstico de câncer, enquanto que 3,46\% afirmaram não possuir nenhum tipo de patologia. Quanto ao uso de medicação, 76,92\% relataram fazer uso contínuo de um ou mais medicamentos, enquanto que 23,07\% disseram não necessitar de nenhum tipo de medicação para sua vida diária (Tabela 3).

No questionamento referente à autopercepção dos idosos quanto a sua saúde, observou-se que 
6,53\% definiram sua saúde ótima, 49,33\% consideraram sua saúde boa, 33,46\% julgaram regular, 7,30\% ruim e apenas 3,46\% como péssima. Quando comparavam sua saúde com alguém da mesma idade, $6,53 \%$ dos idosos julgaram estar com a saúde ótima, 49,61\% consideraram estar com a saúde boa, $33,46 \%$ regular, $6,92 \%$ ruim e $3,84 \%$ péssima. Comparando a saúde atual com 12 meses atrás, $51,53 \%$ relataram estar com a saúde igual, $26,53 \%$ deles julgaram estar melhor, $21,53 \%$ deles consideraram estar pior e apenas $0,38 \%$ consideraram sua saúde como péssima (Tabela 4).

A ocorrência de quedas e suas consequências também foi alvo deste estudo, em que $70,77 \%$ dos idosos pesquisados não apresentaram histórico de quedas, enquanto que $29,23 \%$ dos idosos relataram ter sofrido pelo menos uma queda no último ano. Dentre as consequências deste evento, $53,95 \%$ apresentaram pequenos machucados (PM), 19,74\% sofreram fraturas fechadas (FF) e 26,32\% não apresentaram ferimentos (SM). Dos 41 idosos que sofreram algum tipo de machucado, 7 foram na cabeça/pescoço, 6 na coluna, 6 nas costelas, 14 em membros inferiores e 7 em membros superiores. Quanto ao número de fraturas, dois sujeitos apresentaram fraturas em costelas, oito em membros inferiores e cinco em membros superiores (Tabela 5).

\section{Discussão}

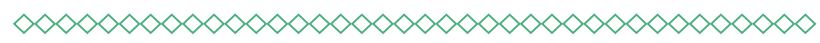

Para os próximos vinte anos, a previsão é de que os brasileiros idosos serão aproximadamente $15 \%$ do total da população, enquanto que no município de Constantina (RS) este número já foi alcançado em $2007^{1}$. 0 município figura como uma cidade longeva e que possui particularidades que estão garantindo um número expressivo de idosos no meio rural, devido ao êxodo dos jovens para buscar trabalho no meio urbano, o que aumenta a proporção de idosos em relação ao número de habitantes inseridos nas demais faixas etárias, ou porque efetivamente os idosos estão vivendo mais.

Mais da metade da amostra era composta por mulheres (56,53\%), dados confirmados por outros estudos e pesquisas realizados com base populacional sobre o envelhecimento humano ${ }^{8}$. No Brasil para cada 100 mulheres idosas existem 78,8 homens (da população total, área urbana e rural) ${ }^{1}$.

A feminização é hoje uma característica da população idosa. Como exemplo, em 1995, de um total de 7,7 milhões de idosos existentes no País, em torno de $55 \%$ eram mulheres, ou seja, para cada 10 idosos havia cerca de 10 idosas. Em projeções elaboradas para 2050, o percentual de contingente feminino acima de 65 anos chegaria a $58,4 \%$ dos 38,3 milhões de idosos estimados, o que implica em 14 idosas para cada 10 idosos. A ampliação da população idosa feminina, com uma taxa média de crescimento geométrico de $3 \%$ ao ano para o período de 1995 a 2050 ocorreria em taxas mais elevadas do que as projetadas para a população idosa masculina, de crescimento no mesmo intervalo de tempo de $2,8 \%$ ao ano $^{9}$. A predominância da população feminina entre os idosos é comprovada internacionalmente e é maior nos países desenvolvi$\operatorname{dos}^{10}$. A maior longevidade do sexo feminino pode estar relacionada com os hábitos e cultura, pois a mulher cuida mais da própria saúde e o homem possui menor propensão em buscar auxílio médico. Outro fator que pode influenciar nesta diferença é a maior divulgação e existência de programas relacionados à saúde da mulher, pois somente em 27 de agosto de 2009 o Ministério da Saúde lançou a Política Nacional de Saúde do Homem com programas voltados para a prevenção e promoção da saúde masculina ${ }^{11}$.

Os resultados mostraram também que a prática de atividade física mais realizada pelos idosos foi à caminhada para 50,38\% dos entrevistados. 0 caminhar está relacionado com as atividades desenvolvidas no viver rural, trabalho no ambiente externo, cuidado de animais (transporte de comida e água), plantio de verduras e legumes, fator relevante para uma percepção positiva de saúde, pois mesmo com o passar dos anos estes idosos mantêm uma atividade laboral, um papel na estrutura da família, e sentem-se funcionais em relação àqueles que se aposentam no ambiente urbano e não podem continuar a exercer seu trabalho.

Apresentou-se neste estudo um número significativo de idosos que não realizam atividade física, $42,30 \%$. Dentre os fatores mais comuns estão às dificuldades na realização de determinadas atividades físicas devido a limitações funcionais, não 
possuir hábito e ter pouco incentivo para sua prática ${ }^{12}$. É importante destacar que diversos estudos realizados ${ }^{13,14,15}$ tem demonstrado que a atividade física possui efeitos benéficos para o prolongamento dos anos de vida, com maior qualidade e autonomia. Dessa maneira, percebe-se a necessidade de se implantar programas de incentivo a prática regular de exercícios e atividades físicas nesse meio, já que há porcentagem importante de idosos que não realizam essas atividades.

As doenças mais comuns relacionadas ao processo de envelhecimento estão fortemente relacionadas às condições de vida ${ }^{16}$. Desta forma, os idosos que residem no ambiente rural são mais vulneráveis a problemas de saúde devido à sobrecarga sofrida ao longo dos anos pela utilização da força excessiva na realização do trabalho que é todo manual, maior quantidade de ingestão de álcool e menor acesso aos serviços de saúde, que pode ser pela distância ou pelo custo ${ }^{17}$.

Neste estudo observou-se que $46,92 \%$ dos idosos são portadores de doenças crônico-degenerativas, destacando o déficit visual (72,30\%) e a doma $(59,23 \%)$, que podem impactar na independência do idoso. Já a hipertensão arterial foi citada por $46,92 \%$ da população do estudo, confirmando ser esta uma enfermidade comum na vida dos idosos, porém, ela não impacta negativamente na realização das atividades de vida diária, reforçando o paradigma da funcionalidade e da dependência como indicador de saúde mais significativo ${ }^{18}$.

Quanto à percepção de saúde autorrelatada pelos idosos da pesquisa, 49,33\% consideraram sua saúde boa e 33,46\% regular. Na comparação de sua própria saúde com alguém da mesma idade, $49,61 \%$ relataram estar boa e $33,46 \%$ regular, na comparação de sua própria saúde com 12 meses atrás $51,53 \%$ julgaram continuar igual e $26,53 \%$ melhor. A maioria $(76,92 \%)$ relatou fazer uso de medicações contínuas, resultado semelhante ao encontrado em estudo realizado por Dal Pizzol ${ }^{19}$ no município de Carlos Barbosa (RS).

Outro estudo realizado por Guerra ${ }^{20}$ no ambiente rural do Rio Grande do Sul observou que 90\% dos idosos refeririam ter algum problema de saúde, porém com uma autopercepção de saúde boa $(49,23 \%)$, semelhante à encontrada neste estudo. Os resultados dessa pesquisa indicam que mesmo o idoso sendo portador de uma ou mais doenças crônicas, quando controladas, continuam sentindo-se saudáveis. Estes dados se diferenciam do estudo realizado por Morais ${ }^{8}$ com idosos acima de 80 anos no município de Encruzilhada do Sul (RS), onde a predominância foi de saúde regular em $46,70 \%$ e saúde boa em $32,80 \%$.

Os idosos residentes em ambiente rural são mais suscetíveis a sofrerem algum tipo de quedas, devido às situações encontradas no próprio meio $^{10}$. As alterações do equilíbrio em idosos são problemas comuns que resultam em importantes limitações na execução das atividades da vida diária e resultam na principal causa risco aumentado de quedas nestes indivíduos ${ }^{19}$.

Na pesquisa realizada, 76 idosos $(29,23 \%)$ relataram ter sofrido quedas no último ano, sendo que destes 41 apresentaram pequenos machucados e somente 15 sofreram algum tipo de fraturas. Estudos realizados por Ribeiro ${ }^{20}$ constataram que as consequências mais citadas das quedas são em primeiro lugar as fraturas, em segundo o medo de cair, após o abandono de atividades, depois a modificação de hábitos e por último a imobilização. É nítida a influência das quedas na qualidade de vida dos idosos, principalmente no que se refere às atividades da vida diária.

Portanto, é importante considerar o nível de funcionalidade do idoso e tentar estimular a manutenção da independência pelo maior tempo possível $^{21}$. A queda, muitas vezes, é sinônima de incapacidade e dependência para o idoso, o que pode demandar maior nível de cuidado, interferindo significativamente não somente na sua saúde, como do cuidador. No estudo, o impacto do evento quedas não foi tão evidente porque poucos resultaram em fratura e a maioria teve somente lesões leves.

Sabendo que o número de idosos vem aumentando significativamente no Brasil, há também a necessidade de melhorar os estudos sobre enveIhecimento humano no contexto rural, ambiente que não tem sido foco de pesquisas. São poucos os estudos em relação aos contextos rurais e é importante modificar esta situação, não só devido ao significativo número de idosos que envelhece em ambientes rurais, mas também porque este cenário possui características específicas que ditam de forma única à experiência de envelhecer ${ }^{22}$. 


\section{Conclusão}

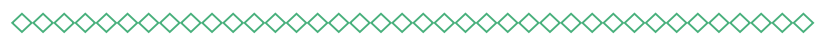

0 aumento significativo do número de idosos é uma realidade tanto para países desenvolvidos quanto para aqueles em desenvolvimento. No caso do Brasil este processo se configura como um grande desafio para a saúde pública, considerando a dimensão territorial deste país, as diferenças de região para região, contextos, culturas e histórias de viver e envelhecer. Este estudo verificou os hábitos de vida, enfermidades e as quedas referidas por idosos residentes no ambiente rural e concluiu-se que a atividade física mais praticada foi a caminhada (50,38\%); a maioria não faz uso de tabaco $(88,16 \%)$ e não ingere bebidas alcoólicas (71,92\%).

Quanto às enfermidades autorreferidas, destacam-se o déficit visual (72,30\%), os distúrbios osteomioarticulares $(59,23 \%)$, hipertensão $(46,92 \%)$ e insônia (41,53\%). No que tange à incidência de quedas, 76 idosos $(29,23 \%)$ relataram ter sofrido queda no último ano.

Em relação à atividade física os achados preocupam, já que praticamente $50 \%$ dos idosos relataram não realizar, principalmente, em função de que a saúde do idoso está vinculada a bons níveis de independência, portanto, a prática regular tanto de exercícios, quanto de atividades físicas são fundamentais.

Destaca-se aqui a importância da continuidade de pesquisas e estudos na área do envelhecimento humano no meio rural, buscando mais dados e subsídios sobre essa realidade a fim de que se possa planejar ações adequadas para essa população com vistas ao envelhecimento saudável e bem-sucedido, prevenindo as doenças crônicas e as limitações físicas próprias do processo de envelhecer.

\section{Referências}

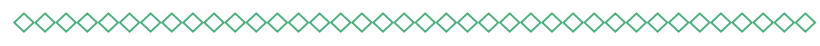

1. Instituto Brasileiro de Geografia e Estatística. Censo demográfico 2010. [Acesso em: 14/07/2013]. Disponivel em: http:// saladeimprensa.ibge.gov.br/noticias?view= noticia\&id=1\&busca $=1$ \&idnoticia $=1866$.
2. Vandervoot AA. Alterações biológicas e fisiológicas. In: Pickles B, Compton A, Cott C, Simpson J, Vandervoot A. Fisioterapia na Terceira idade. 2a ed. São Paulo: Santos; 2000.

3. Sheppard RJ. Envelhecimento, atividade física e saúde. 1a ed. São Paulo: Phorte; 2003.

4. Camargos MC, Machado CJ, Rodrigues RN. A relação entre renda e morar sozinho para idosos paulistanos - 2000. Revista Brasileira Estatística Populacional. 2007;24(1):37-51.

5. Siqueira A, Silva MN. O bem estar da pessoa idosa em meio rural. Análise psicológica. 2002;20(3):505-16.

6. Instituto Brasileiro de Geografia e Estatística. Contagem populacional 2007. [Acesso em: 15/10/2009]. Disponível em: http://www.ibge.gov. br.

7. Crum RM, Anthony JC, Bassett SS, Folstein MF. Population based norms for the mini-mental state examination by age and educational level. JAMA. 1993;269:2386-91.

8. Morais EP. Envelhecimento no meio rural: condições de vida, saúde e apoio aos idosos mais velhos de Encruzilhada do Sul-RS [tese]. Ribeirão Preto: Universidade de São Paulo, Escola de Enfermagem de Ribeirão Preto; 2007.

9. Morais EP et al. Os idosos mais velhos no meio rural: realidade de vida e saúde de uma população do interior gaúcho. Texto \& Contexto Enfermagem. 2008;17(2):374-83.

10. Morais MM. Envelhecimento da população brasileira [tese]. Belo Horizonte: Universidade Federal Minas Gerais, CEDEPLAR; 1997.

11. Kinsella K, Gist YJ. Older workers, retirement, and pensions: a comparative international chartbook. U.S.: Departament of Commerce; Dec., 1995.

12. BRASIL. Ministério da Saúde. Portaria $n^{\circ} 1.944$ de 27 de agosto de 2009. [Acesso em: 14/07/2013]. Disponível em: http://bvsms. saude.gov.br/bvs/saudelegis/gm/2009/ prt1944_27_08_2009.html. 
13. Anderson MIP. Saúde e condições de vida do idoso no Brasil [dissertação]. Rio de Janeiro: Universidade do Estado do Rio de Janeiro, Instituto de Medicina Social, 1997.

14. Matsudo SM, Matsudo VKR. Exercício, densidade óssea e osteoporose. Rev Bras Ortop. 1992;27(10):730-42.

\section{Sharkey BJ. Condicionamento Físico e} Saúde. 4a ed. Porto Alegre: Artmed; 1998.

16. Nieman D. Exercício e saúde: Como se prevenir de doenças usando o exercício como seu medicamento. São Paulo: Manole; 1999.

\section{Saldanha AL, Caldas CP. Saúde do Idoso: A} arte de Cuidar. 2a ed. Rio de Janeiro; 2004.

18. Silva OJ. Exercício em situações especiais 1: Crescimento, Flexibilidade, alterações posturais, asmas, diabetes, terceira idade. Florianopolis: Ed UFSC; 1997.
19. Francisco CM. Estudos dos fatores preditores de envelhecimento sem incapacidade funcional entre idosos de velhice avançada no município de São Paulo [dissertação]. São Paulo: Universidade de São Paulo, Escola de Enfermagem; 2006.

20. Dal Pizzol TS, Pons ES, Hugo FN, Bozzetti MC, Sousa MLR, Hilgert JB. Uso de medicamentos entre idosos residentes em áreas urbanas e rurais de município no Sul do Brasil: um estudo de base populacional. Cad. Saúde. 2012;28(1):104-14.

21. Guerra RO, Maciel ACC. Prevalência e fatores associados ao déficit de equilíbrio em idosos. RBCM. 2005;13(1):37-44.

22. Ribeiro AP, Souza ER, Atie S, Souza AC, Schilithz AO. A influência das quedas na qualidade de vida de idosos. Ciências da saúde coletiva. 2008;13(4):1265-73.

\section{Anexos}

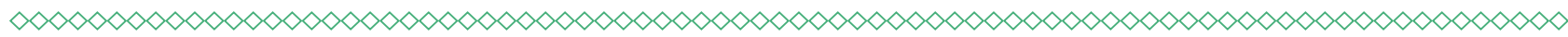

Tabela 1 - Distribuição etária dos idosos em território rural no município de Constantina (RS)

\begin{tabular}{ccc} 
Idade & $n$ & $\%$ \\
60 a 69 anos & 112 & 43,07 \\
70 a 79 anos & 111 & 42,70 \\
80 a 89 anos & 26 & 10,00 \\
Acima de 90 anos & 11 & 4,23 \\
\hline
\end{tabular}

Fonte: elaboração dos autores.

(clique para voltar ao texto)

Tabela 2 - Hábitos de vida dos idosos residentes no ambiente rural de Constantina (RS), em 2010

\begin{tabular}{|c|c|c|c|}
\hline \multicolumn{2}{|c|}{ Hábitos de vida } & $\mathrm{n}$ & $\%$ \\
\hline Atividade física & $\begin{array}{l}\text { Caminhada } \\
\text { Jogo de bocha } \\
\text { Jogo de futebol } \\
\text { Não pratica }\end{array}$ & $\begin{array}{c}131 \\
17 \\
2 \\
110\end{array}$ & $\begin{array}{c}50,38 \\
6,53 \\
0,76 \\
42,30\end{array}$ \\
\hline Tabagismo & $\begin{array}{l}\text { Não fumante } \\
\text { Fumante }\end{array}$ & $\begin{array}{c}231 \\
29\end{array}$ & $\begin{array}{l}88,16 \\
11,16\end{array}$ \\
\hline Bebida alcóolica & $\begin{array}{l}\text { Não usuário } \\
\text { Usuário }\end{array}$ & $\begin{array}{c}187 \\
73\end{array}$ & $\begin{array}{l}71,92 \\
28,07\end{array}$ \\
\hline
\end{tabular}


Tabela 3 - Enfermidades e medicação autorreferidas pelos idosos do ambiente rural do município de Constantina (RS), em 2010

\begin{tabular}{lcccc} 
Enfermidade & \multicolumn{2}{c}{ Refere } & \multicolumn{2}{c}{ Não refere } \\
Déficit de visão & $n$ & $\%$ & 72 & 27,70 \\
DOMA & 188 & 72,30 & 115 & 40,77 \\
Hipertensão & 145 & 59,23 & 138 & 53,08 \\
Insônia & 122 & 46,92 & 152 & 58,47 \\
Tonturas & 108 & 41,53 & 198 & 76,16 \\
Incontinência urinária & 62 & 23,84 & 205 & 78,85 \\
Déficit de audição & 55 & 21,15 & 209 & 80,39 \\
Bronquite asmática & 51 & 19,61 & 228 & 87,70 \\
Depressão & 32 & 12,30 & 236 & 90,77 \\
Câncer & 24 & 9,23 & 250 & 96,16 \\
Não apresentam patologia & 10 & 3,84 & 291 & 96,54 \\
Fazem uso de medicação & 9 & 3,46 & 60 & 23,07 \\
\hline
\end{tabular}

Fonte: elaboração dos autores.

(clique para voltar ao texto)

Tabela 4 - Percepção da saúde relatada pelos idosos do ambiente rural do município de Constantina (RS), em 2010

\begin{tabular}{llcc} 
& & $n$ & $\%$ \\
& Ótima & 17 & 6,53 \\
Auto-percepção de saúde & Boa & 128 & 49,23 \\
& Regular & 87 & 33,46 \\
& Ruim & 19 & 7,3 \\
& Péssima & 9 & 3,46 \\
& Ótima & 16 & 6,15 \\
& Boa & 129 & 49,61 \\
& Regular & 87 & 33,46 \\
& Ruim & 18 & 6,92 \\
& Péssima & 10 & 3,84 \\
& Igual & 134 & 51,53 \\
& Melhor & 69 & 26,53 \\
& Pior & 56 & 21,53 \\
& Péssima & 1 & 0,38 \\
\hline
\end{tabular}

Fonte: elaboração dos autores.

(clique para voltar ao texto) 
Tabela 5 - Ocorrência de quedas relatada pelos idosos do ambiente rural do município de Constantina (RS), em 2010

\begin{tabular}{|c|c|c|c|c|c|c|c|c|c|c|c|}
\hline \multirow{2}{*}{ Quedas } & \multicolumn{3}{|c|}{ Consequência } & \multicolumn{5}{|c|}{ Local de ferimento } & \multicolumn{3}{|c|}{ Fratura } \\
\hline & PM & $\mathrm{FF}$ & SM & $\mathrm{CP}$ & C & $\mathrm{CT}$ & MI & MS & $\mathrm{CT}$ & MI & MS \\
\hline$n=76$ & 41 & 15 & 20 & 7 & 6 & 6 & 14 & 7 & 2 & 8 & 5 \\
\hline
\end{tabular}

Legenda: PM: Pequenos machucados. FF: Fratura fechada. SM: Sem machucado. CP: Cabeça e pescoço. C: Coluna. CT: Costelas. MI: Membros inferiores. MS: Membros superiores. 